\section{Reclassificação dos grupos de causas prováveis dos óbitos de causa mal definida, com base nas Autorizações de Internação Hospitalar no Sistema Único de Saúde, Estado do Rio de Janeiro, Brasil}

\author{
Probable cause of death after reclassification \\ of ill-defined causes on hospital admissions \\ forms in the Unified National Health System, \\ Rio de Janeiro, Brazil
}

Claudio Luiz dos Santos Teixeira 1 Carlos Henrique Klein 2

Kátia Vergetti Bloch 1

Claudia Medina Coeli 1
1 Núcleo de Estudos de Saúde Coletiva, Universidade Federal do Rio de Janeiro Rio de Janeiro, Brasil.

2 Escola Nacional de Saúde Pública Sergio Arouca, Fundação Oswaldo Cruz, Rio de Janeiro, Brasil.

Correspondência C. L. S. Teixeira Núcleo de Estudos de Saúde Coletiva, Universidade Federal do Rio de Janeiro. Rua Anfilóquio de Lima 78 Campos, $R J$

28051-050, Brasil.

cteixeira@fmc.br

\section{Abstract}

This article describes the clinical and socio-demographic characteristics of deaths from ill-defined causes in the State of Rio de Janeiro, Brazil, in 1998, and reclassifies the groups of probable causes based on data from death certificates in the Mortality Information System and the Hospital Information System of the Unified National Health System (SIH-SUS) for 1997-98. Reclassification required a random sample of defined causes from the SIH-SUS. The technique used was probabilistic relationship of records. As compared to deaths with defined causes, in those with ill-defined causes the individuals were predominantly non-white, had less schooling, had died in Greater Metropolitan Rio de Janeiro, were less likely to have been hospitalized in the SUS, and were more likely to have died at home and without medical care. It was possible to reclassify 20\% of deaths with ill-defined causes. Reclassification had a minor impact on proportional mortality, due to the size of this group (10\% of the deaths). However, if the results could be applied to all deaths with ill-defined causes, the impact might be greater on proportional mortality.

Information Systems; Mortality Registries; Death Certificates

\section{Introdução}

A proporção de óbitos de causa mal definida ou causa ignorada, entre todos os óbitos ocorridos, tem sido o indicador mais utilizado para avaliar a qualidade da informação sobre causa de morte. Elevada proporção de óbitos de causa mal definida indica que os dados são de qualidade duvidosa, além de subestimar outras causas de morte. No entanto, um valor reduzido para este indicador não significa, necessariamente, boa qualidade da informação 1 .

Óbito de causa mal definida ou de causa ignorada é aquele em que as causas de morte não foram definidas na Declaração de Óbito (DO) porque o falecido não teve assistência médica suficiente para identificar a causa do óbito, ou chegou já cadáver à unidade de saúde, ou, ainda, porque $o$ atestante omitiu informações.

Apesar de, no Brasil como um todo, haver uma tendência de queda da proporção de óbitos de causa mal definida, reduzindo de $15,1 \%$ em 1999 para 14,3\% em 2000 2, o mesmo não ocorre na Região Metropolitana do Rio de Janeiro. Em 1980, a proporção de óbitos de causa mal definida foi de $2,1 \%$, passando para $4,9 \%$ em 1990 e chegando a 10,4\% em 1995 3, estabilizando acima de $10 \% 4$.

Esse crescimento coincide com a publicação da Portaria n. 550 pela Secretaria Estadual de Saúde do Rio de Janeiro (SES/RJ), em janeiro de 1990 , ainda em vigor, que determina em 
seu Artigo 2o que "esgotadas todas as tentativas de se determinar a causa básica da morte e não havendo suspeita de óbito por causa violenta, deverá ser declarado na parte I do atestado médico Causa Indeterminada" 5 (parte I, p. 19).

A existência de registros hospitalares, organizados na grande base de dados do Sistema de Informações Hospitalares do Sistema Único de Saúde (SIH-SUS), oferece a possibilidade de recuperar, mesmo que parcialmente, as possíveis causas dos óbitos de causa mal definida, tarefa provavelmente não realizada pelos sistemas oficiais de estatísticas de óbitos. Entretanto, no Estado do Rio de Janeiro, onde a ocorrência de óbitos de causa mal definida é bastante elevada há mais de uma década, torna-se necessário empreender esforço adicional na reclassificação das causas dos óbitos de causa mal definida. Essa medida visa não prejudicar as análises de séries históricas das taxas de mortalidade pelas causas definidas. Além disso, o melhor conhecimento dos fatores associados à identificação da causa de óbito pode subsidiar ações direcionadas à melhoria da qualidade da informação.

É objetivo do presente estudo descrever os óbitos de causa mal definida ocorridos no Estado do Rio de Janeiro, em 1998, de acordo com informações obtidas nas DO, e também reclassificar as possíveis causas de óbito deste grupo, de acordo com os diagnósticos registrados no SIH-SUS referentes às internações ocorridas até um ano antes dos óbitos.

\section{Material e métodos}

Os dados deste estudo foram fornecidos pela SES/RJ e o DATASUS (Departamento de Informação e Informática do SUS), e correspondem aos dados de 1998 do Sistema de Informação sobre Mortalidade (SIM) e aos dados de $1997 \mathrm{e}$ 1998 do SIH-SUS.

Selecionaram-se no SIM os óbitos classificados como de causas mal definidas, totalizando, em 1998, 12.633 óbitos (10,5\% de todos os óbitos ocorridos no Estado do Rio de Janeiro). Foram excluídos os registros em que não constava o nome do indivíduo, restando $10.692 \mathrm{ca}$ sos de óbitos de causa mal definida para relacionamento com os dados do SIH-SUS. Não foram incluídos os óbitos fetais.

Com a finalidade de comparar os relacionamentos, de óbitos mal definidos com as Autorizações de Internação Hospitalar (AIH) com aqueles formados por óbitos definidos com os mesmos tipos de dados do SIH-SUS, foi selecionada uma amostra aleatória de mesmo tamanho, sem reposição, de 10.692 óbitos, entre aqueles de causas definidas, também ocorridos no ano de 1998.

Empregou-se o relacionamento probabilístico dos registros de óbitos (óbitos de causa mal definida e amostra de óbitos de causa definida) no SIM, para o pareamento com os registros do SIH-SUS de 1997 e 1998. Foram excluídos os registros do SIH-SUS em que o período entre a data de saída do hospital e a data do óbito excedeu 365 dias, além dos óbitos fetais. Foram recuperadas no SIH-SUS informações de 5.385 pacientes referentes a 9.407 internações. Destes, 2.133 referem-se aos óbitos de causa mal definida e 3.252 aos óbitos de causa definida.

Na utilização do método probabilístico de relacionamento de banco de dados para concatenar os dados do SIM com os dados do SIHSUS foram executados os seguintes processos: (1) rotinas para padronização do formato das variáveis dos bancos; (2) blocagem, que consistiu na criação de conjuntos comuns de registros de acordo com a chave de identificação; (3) aplicação de algoritmos para comparações entre campos (ex. comparação aproximada de cadeias de caracteres); (4) cálculo de escores, que resumiram o grau de concordância global entre registros de um mesmo par; (5) definição de limiares para o relacionamento dos pares de registros relacionados em verdadeiros, duvidosos e não pares; (6) revisão manual dos pares duvidosos, visando à reclassificação dos mesmos como pares verdadeiros ou não pares. Descrição detalhada do processo de relacionamento foi feita por Teixeira 6 e Teixeira et al. 7 .

Foi escolhido o RecLinkII versão 2.1.7 (http: //paginas.terra.com.br/educacao/kencamargo/RecLinkII. html, acessado em 06/Jul/2003), para a aplicação do método de relacionamento por tratar-se de um programa desenvolvido especificamente para tal fim e já utilizado no tratamento de outros bancos de dados 8,9,10,11,12.

As variáveis data do óbito, sexo, idade, estado civil, escolaridade, cor da pele, local de ocorrência do óbito, identificação de quem notificou, recebimento ou não de assistência médica, unidade de saúde e causa básica do óbito foram obtidas nas DO. Data da saída, data da internação, procedimentos e diagnóstico principal da internação originaram-se do SIH-SUS.

O diagnóstico principal selecionado nos registros do SIH-SUS referiu-se à última internação do caso de óbito registrado no SIM. Caso este fosse indeterminado, utilizou-se o dado do campo do procedimento realizado. Na impossibilidade de identificação do diagnóstico, o registro foi classificado como óbito de causa mal definida. Os diagnósticos foram agrupados por capítulos da CID-10 (Classificação Estatística 
Internacional de Doenças e Problemas Relacionados à Saúde) 13. Os diagnósticos do SIH-SUS classificados nas seções "Lesões" e "Fatores Externos" foram equiparados ao capítulo de "Causas Externas” de causas de óbito nas DO.

Com base na informação do "diagnóstico principal” (SIH-SUS), criou-se um índice de correção, descrito adiante, para estimar a probabilidade de que a causa do óbito da causa mal definida seja a mesma indicada no diagnóstico principal no SIH-SUS. Pautado nos dados de diagnóstico principal no SIH-SUS da amostra de óbitos de causa definida obtida do SIM, calculou-se a probabilidade de o óbito ocorrer por cada capítulo da CID-10. Exemplificando: entre os óbitos de causa definida com causa básica "Doença do Aparelho Circulatório”, observaram-se 645 óbitos no SIM e 916 registros no SIH-SUS com diagnóstico principal no capítulo da "Doença do Aparelho Circulatório”. A probabilidade de óbitos de causas definidas por "Doença do Aparelho Circulatório" entre os registros do SIH-SUS com diagnóstico de "Doença do Aparelho Circulatório" foi calculada em 0,7041 (645/916). O mesmo procedimento foi realizado para todos os capítulos da CID-10. A probabilidade para cada capítulo foi multiplicada pelo número de óbitos de causa mal definida no SIM com diagnóstico principal no SIH-SUS no respectivo capítulo da CID10. A soma dos produtos correspondeu à quantidade provável de óbitos por "Doença do Aparelho Circulatório”, por exemplo: (669 SIH com "Doença do Aparelho Circulatório" x 0,7041 = $471)+(361$ SIH com "Doença do Aparelho Respiratório” x 0,2695 = 95) + (52 SIH-SUS com "Doenças do Sistema Nervoso" x 0,57 = 30), e assim por diante, de forma que, somando-se os produtos para os 18 capítulos da CID-10 encontrados, obteve-se a quantidade total de óbitos por “Doença do Aparelho Circulatório” = 772 .

As análises estatísticas foram realizadas com os programas Epi Info versão 6.04 (Centers for Disease Control and Prevention, Atlanta, Estados Unidos) e Stata 7.0 (Stata Corporation, College Station, Estados Unidos). Para a amostra de óbitos de causa definida relacionados, foram calculados os intervalos de confiança de 95\% (IC95\%) das estimativas dos percentuais dos atributos sócio-demográficos, local do óbito e serviço que notificou o óbito. Para os óbitos de causa mal definida, por se tratarem de dados populacionais, não foram calculados intervalos de confiança.

Para o cálculo da taxa de mortalidade proporcional por causa, utilizaram-se dados do DATASUS do mesmo ano 14. Foi calculado o coeficiente kappa na análise de concordância entre o diagnóstico da causa básica do óbito declarada na DO e o diagnóstico principal registrado na SIH-SUS. O projeto foi aprovado pelo Comitê de Ética em Pesquisa do Núcleo de Estudos de Saúde Coletiva, Universidade Federal do Rio de Janeiro em 26 de junho de 2003 (processo n. 029/2003).

\section{Resultados}

Entre os 12.633 casos de óbitos de causa mal definida no SIM, 84,6\% foram classificados como "outras causas mal definidas e as não especificadas de mortalidade" (CID-10, R99); 11,7\%, como "sintomas e sinais relativos aos aparelhos e demais sintomas e sinais gerais" (CID-10, R00 até CID-10, R68); 3,7\% como "causas mal definidas e desconhecidas de mortalidade" (CID-10, R95 até CID-10, R98).

Nas comparações de variáveis obtidas da DO, na totalidade dos registros e naqueles pareados com registros do SIH-SUS, entre óbitos de causa mal definida e óbitos de causa definida, observou-se que o percentual de mulheres nos óbitos de causa mal definida foi maior do que nos óbitos de causa definida. Em relação à idade, não se percebeu diferença importante entre os casos de óbitos de causa mal definida e óbitos de causa definida, além de um discreto predomínio dos óbitos de causa mal definida na faixa de 40 a 69 anos de idade. Quanto à escolaridade, o percentual de casos de óbitos de causa mal definida com segundo grau ou curso superior é a metade dos casos de óbitos de causa definida. Pessoas de cor da pele não branca foram relativamente mais freqüentes entre os óbitos de causa mal definida. A maioria dos óbitos ocorreu em hospital, tanto os de causa mal definida, quanto os de causa definida. Nos óbitos de causa mal definida, o percentual dos que ocorreram nos domicílios foi quase o dobro do percentual encontrado para os óbitos de causa definida. O percentual de casos em que o médico ou um substituto identificou o óbito foi menor entre os óbitos de causa mal definida do que entre os de causa definida (Tabela 1).

Com exceção da idade, para todas as demais variáveis, os percentuais das categorias nos óbitos de causa mal definida relacionados foram significativamente diferentes, com grau de confiança de 95\%, daqueles da amostra dos óbitos de causa definida.

Somente $73,3 \%$ das DO tinham informação sobre assistência médica registrada na declaração. Receberam assistência 54,8\% dos casos de óbitos de causa mal definida e o mesmo ocorreu em $85,1 \%$ dos óbitos de causa definida. O estado civil dos casos apresentou distribuições 
Tabela 1

Distribuição, em percentuais, de todos os óbitos de causas mal definidas e de causas definidas, e dos óbitos relacionados (Declaração de Óbito-Autorização de Informação Hospitalar; DO-AlH), segundo características sócio-demográficas e dos óbitos. Estado do Rio de Janeiro, Brasil, 1998.

\begin{tabular}{|c|c|c|c|c|c|}
\hline \multirow[t]{2}{*}{ Características } & \multicolumn{2}{|c|}{ Todos } & \multicolumn{3}{|c|}{ Relacionados } \\
\hline & $\begin{array}{l}\text { Óbitos de causas } \\
\text { mal definidas }\end{array}$ & $\begin{array}{c}\text { Óbitos de causas } \\
\text { definidas }\end{array}$ & $\begin{array}{l}\text { Óbitos de causas } \\
\text { mal definidas* }\end{array}$ & $\begin{array}{l}\text { Óbitos de causas } \\
\text { definidas* }\end{array}$ & IC95\%** \\
\hline Sexo (N) & 12.618 & 102.574 & 2.133 & 3.252 & \\
\hline Masculino & 53,2 & 58,0 & 53,0 & 57,3 & $55,6-59,1$ \\
\hline Feminino & 46,8 & 42,0 & 47,0 & 42,7 & $40,9-44,4$ \\
\hline Total & 100,0 & 100,0 & 100,0 & 100,0 & \\
\hline Idade em anos $(\mathrm{N})$ & 12.226 & 99.228 & 2.133 & 3.252 & \\
\hline $0-39$ & 14,6 & 21,0 & 14,4 & 15,3 & $14,1-16,6$ \\
\hline $40-69$ & 46,2 & 39,1 & 45,6 & 45,2 & $43,5-47,0$ \\
\hline 70 ou mais & 39,2 & 39,9 & 40,0 & 39,5 & $37,8-41,2$ \\
\hline Total & 100,0 & 100,0 & 100,0 & 100,0 & \\
\hline Escolaridade $(\mathrm{N})$ & 7.647 & 62.130 & 1.302 & 1.941 & \\
\hline Nenhuma e 1ㅇ grau & 90,5 & 80,8 & 94,3 & 89,4 & $87,9-90,7$ \\
\hline 2o grau e superior & 9,5 & 19,2 & 5,7 & 10,6 & $9,3-12,1$ \\
\hline Total & 100,0 & 100,0 & 100,0 & 100,0 & \\
\hline Cor da pele (N) & 10.567 & 87.468 & 1.794 & 2.746 & \\
\hline Branca & 52,0 & 60,9 & 50,1 & 56,4 & $54,5-58,2$ \\
\hline Não branca & 48,0 & 39,1 & 49,9 & 43,6 & $41,8-45,5$ \\
\hline Total & 100,0 & 100,0 & 100,0 & 100,0 & \\
\hline Local do óbito (N) & 12.604 & 102.545 & 2.133 & 3.251 & \\
\hline Hospital & 69,0 & 76,4 & 73,8 & 90,1 & $89,0-91,1$ \\
\hline Via pública & 3,9 & 7,3 & 2,4 & 0,7 & $0,4-1,0$ \\
\hline Domicílio & 23,3 & 14,0 & 21,3 & 8,4 & $7,5-9,4$ \\
\hline Outro & 3,8 & 2,3 & 2,5 & 0,8 & $0,6-1,2$ \\
\hline Total & 100,0 & 100,0 & 100,0 & 100,0 & \\
\hline Quem identificou (N) & 8.893 & 83.259 & 1.431 & 2.577 & \\
\hline Com assistência*** & 41,7 & 64,7 & 47,2 & 73,2 & $71,5-74,9$ \\
\hline Sem assistência & 58,3 & 35,3 & 52,8 & 26,8 & $25,1-28,5$ \\
\hline Total & 100,0 & 100,0 & 100,0 & 100,0 & \\
\hline
\end{tabular}

* Óbitos de causas mal definidas relacionados e amostra de óbitos de causas definidas, $n=10.692$;

** Intervalos de confiança de $95 \%$ na amostra de óbitos de causas definidas;

*** Com assistência: médico que atendeu o paciente ou substituto.

semelhantes nos óbitos de causa mal definida e naqueles de causa definida, no total e nos óbitos relacionados, não sendo, por isso, incluído na Tabela 1 .

O percentual de casos de óbitos no SIM, cujo óbito ocorreu na internação analisada, foi 2,6 vezes maior no grupo dos óbitos de causa definida (Tabela 2).

O tempo médio entre a última internação e o óbito foi de 97 dias nos óbitos de causa mal definida e de 49 dias nos óbitos de causa definida. Entre os óbitos de causa mal definida, ocorreu 1,6 internação por pessoa e, entre os óbitos de causa definida, 1,9. A mediana da duração das internações entre os óbitos de causa mal definida foi de sete dias, variando de 1 a 157, não havendo diferença entre os sexos. Na amostra dos óbitos de causa definida, a mediana foi de sete dias (mínimo 1 e máximo 182), não havendo, como nos óbitos de causa mal definida, discrepância entre os sexos.

As distribuições percentuais, pelas regiões do Estado, dos óbitos de causa definida relacionados, dos óbitos de causa mal definida re- 
Relacionamento Declaração de Óbito-Autorização de Informação Hospitalar (DO-AlH) de óbitos de causas mal definidas e amostra de óbitos de causas definidas, segundo tipo de alta. Estado do Rio de Janeiro, Brasil, 1998.

\begin{tabular}{lccc}
\hline Tipo de alta & $\begin{array}{c}\text { Óbitos de causas mal } \\
\text { definidas }(\mathbf{N}=\mathbf{2 . 1 3 3}) \\
\%\end{array}$ & \multicolumn{2}{c}{$\begin{array}{c}\text { Óbitos de causas } \\
\text { definidas }(\mathbf{N}=\mathbf{3 . 2 5 2}) \\
\%\end{array}$} \\
\hline & $\begin{array}{l}\% \\
\text { IC95\% }\end{array}$ \\
\hline Coincidente com o óbito & 24,4 & 63,7 & $62,0-65,4$ \\
Última internação antes do óbito* & 75,6 & 36,3 & $34,6-38,0$ \\
Total & 100,0 & 100,0 & \\
\hline
\end{tabular}

* Até um ano antes do óbito.

lacionados e da totalidade dos óbitos evidenciam que, na região metropolitana, ocorreram $77,9 \%$ dos óbitos de todo o Estado do Rio de Janeiro. Vale destacar que esta mesma região foi responsável por $83,3 \%$ dos casos de óbitos de causa mal definida, $69,9 \%$ do total de óbitos de causa mal definida relacionados, e $66,9 \%$ da amostra de óbitos de causa definida.

Observa-se que, nas cinco regiões com percentual de óbitos de causa mal definida abaixo de $10 \%$, ocorreram apenas $15 \%$ do total de óbitos do Estado do Rio de Janeiro.

O gráfico da Figura 1 organizou as regiões pelo percentual de óbitos de causa mal definida de maneira crescente. Observa-se que em três regiões, Baixada Litorânea, Norte e Metropolitana, esse percentual é superior a $10 \%$. Quando consideramos o percentual de relacionamentos obtido por região, nota-se que a relação amostra de óbitos de causa definida/óbitos de causa mal definida se mantém, com uma proporção um pouco maior de pares relacionados na amostra de óbitos de causa definida em quase todas as regiões, exceto nas regiões Baía da Ilha Grande e Médio Paraíba, onde essa diferença é substancial.

Percebe-se uma relação entre percentual de óbitos de causa mal definida por região e os óbitos relacionados. As regiões com percentuais maiores de óbitos de causas mal definidas, acima de $10 \%$, possibilitaram, relativamente, menos relacionamentos entre DO e AIH do que aquelas regiões onde ocorreram menores percentuais de óbitos de causas mal definidas.

A Região Noroeste fluminense ilustra bem esse desequilíbrio, embora ocupe o sexto lugar em percentual de óbitos de causa mal definida, foi possível relacionar mais de $65 \%$ da amostra de óbitos de causa definida e mais de $40 \%$ dos óbitos de causa mal definida (Figura 1).

Quanto ao local de ocorrência, foi verificado que a distribuição de todos os casos de óbi- tos de causa mal definida não foi homogênea entre as unidades de saúde. As dez unidades com maior volume de atendimentos e internações que resultaram em óbitos, foram responsáveis por apenas cerca de um quinto de todos os óbitos hospitalares, porém emitiram quase a metade das DO com óbitos de causa mal definida $(48,5 \%)$ (Tabela 3 ).

Foi de 0,45 o coeficiente de concordância kappa 15 entre as causas básicas dos óbitos registrados nas DO, classificadas pelos capítulos da CID-10, com os respectivos diagnósticos principais, anotados nas SIH-SUS. Quando comparados apenas os casos dos cinco capítulos mais freqüentes, esse valor foi de 0,63.

Os resultados das reclassificações das causas de óbito dos óbitos de causa mal definida segundo os capítulos da CID-10 mostram que, entre os óbitos de causa mal definida, as "Doenças do Aparelho Circulatório" são as causas prováveis de óbito mais freqüentes. O grupo de doenças "Endócrinas, Nutricionais e Metabólicas” está mais representado, e o de causas externas, menos, entre os óbitos de causa mal definida do que entre os óbitos de causa definida.

A reclassificação dos óbitos de causa mal definida praticamente não afetou a distribuição da mortalidade proporcional, excluindo-se os óbitos de causa mal definida (Tabela 4).

\section{Discussão}

Apenas uma pequena proporção dos casos de óbitos de causa mal definida ocorridos em 1998 no Estado do Rio de Janeiro pôde ser esclarecida pelo método de relacionamento probabilístico aplicado na conjugação DO/SIH-SUS. Só conseguiram ser reclassificados $16,3 \%$ de todos óbitos de causa mal definida. As alterações na mortalidade proporcional resultantes da reclassificação foram modestas. 
Figura 1

Distribuições por região, em percentuais, dos óbitos de causas mal definidas, óbitos de causas mal definidas relacionados e da amostra de óbitos de causas definidas relacionados. Estado do Rio de Janeiro, Brasil, 1998.

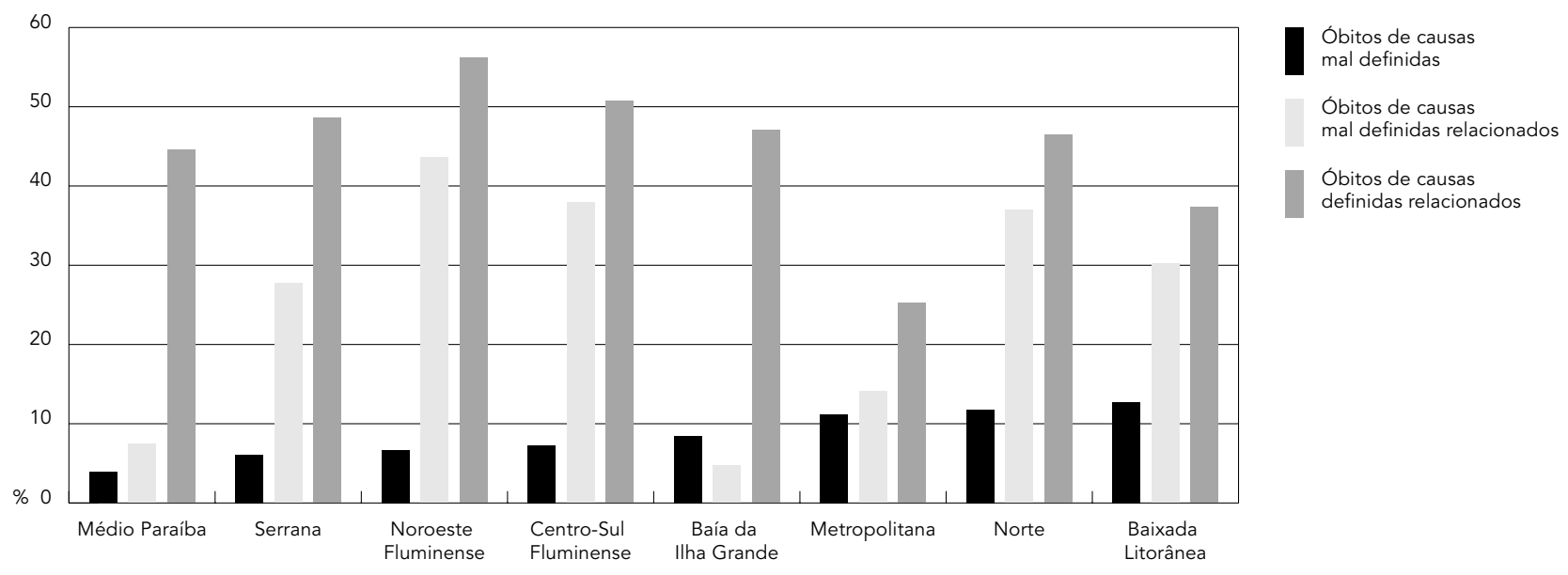

Tabela 3

Distribuições, em percentuais, dos óbitos*, por algumas unidades de saúde no Estado do Rio de Janeiro, Brasil, 1998.

\begin{tabular}{|c|c|c|c|c|c|}
\hline \multirow[t]{2}{*}{ Unidade de saúde (Local) } & \multicolumn{3}{|c|}{ Todos os óbitos } & \multicolumn{2}{|c|}{ Óbitos relacionados } \\
\hline & $\begin{array}{c}\text { Óbitos de causas } \\
\text { mal definidas } \\
(\mathrm{N}=8.555) \\
\%\end{array}$ & $\begin{array}{c}\text { Óbitos de causas } \\
\text { definidas } \\
(N=76.063) \\
\%\end{array}$ & $\begin{array}{c}\text { Total } \\
(\mathrm{N}=84.618) \\
\%\end{array}$ & $\begin{array}{c}\text { Obitos de causas } \\
\text { mal definidas } \\
(\mathrm{N}=1.574) \\
\%\end{array}$ & $\begin{array}{c}\text { Óbitos de causas } \\
\text { definidas } \\
(\mathrm{N}=2.917) \\
\%\end{array}$ \\
\hline $\begin{array}{l}\text { Hospital de Duque de Caxias } \\
\text { (Duque de Caxias) }\end{array}$ & 9,2 & 1,1 & 1,9 & 10,2 & 0,8 \\
\hline Hospital Albert Schweitzer (Realengo) & 7,7 & 1,4 & 2,0 & 6,1 & 1,3 \\
\hline $\begin{array}{l}\text { Hospital Geral de Nova Iguaçu/ } \\
\text { Hospital da Posse (Nova Iguaçu) }\end{array}$ & 5,0 & 3,9 & 4,0 & 3,7 & 2,2 \\
\hline Hospital Carlos Chagas (Marechal Hermes) & 4,6 & 1,4 & 1,7 & 4,1 & 1,3 \\
\hline Hospital Rocha Faria (Campo Grande) & 4,4 & 2,3 & 2,5 & 3,6 & 2,0 \\
\hline $\begin{array}{l}\text { Hospital Municipal Salgado Filho } \\
\text { (Engenho Novo) }\end{array}$ & 4,1 & 2,6 & 2,8 & 3,0 & 1,6 \\
\hline $\begin{array}{l}\text { Posto de Assistência Médica Meriti } \\
\text { (São João de Meriti) }\end{array}$ & 3,9 & 0,6 & 0,9 & 5,0 & 0,7 \\
\hline Hospital Pedro II (Santa Cruz) & 3,8 & 1,3 & 1,6 & 4,9 & 1,3 \\
\hline $\begin{array}{l}\text { Pronto de Socorro Dr. Armando } \\
\text { Gomes Couto (São Gonçalo) }\end{array}$ & 2,9 & 0,9 & 1,1 & 0,0 & 0,0 \\
\hline $\begin{array}{l}\text { Hospital Municipal Juscelino } \\
\text { Kubistchek (Nilópolis) }\end{array}$ & 2,9 & 1,1 & 1,3 & 1,5 & 0,3 \\
\hline Outras 713 unidades & 51,5 & 83,4 & 80,2 & 57,8 & 88,4 \\
\hline Total & 100,0 & 100,0 & 100,0 & 100,0 & 100,0 \\
\hline
\end{tabular}

* Óbitos cujo local de ocorrência foram registrados como ocorridos em unidades de saúde. 
Mortalidade proporcional (\%) por causas de óbito (CID-10 13), antes e após a reclassificação dos óbitos de causas mal definidas relacionados com Autorização de Informação Hospitalar (AlH) no Estado Rio de Janeiro, Brasil, 1998.

\begin{tabular}{|c|c|c|c|c|c|c|}
\hline $\begin{array}{l}\text { Capítulo } \\
\text { da CID-10 }\end{array}$ & $\begin{array}{l}\text { Número geral } \\
\text { de óbitos }(n)\end{array}$ & $\begin{array}{l}\text { Mortalidade } \\
\text { proporcional (\%) }\end{array}$ & $\begin{array}{l}\text { Mortalidade } \\
\text { proporcional } \\
\text { sem causas mal } \\
\text { definidas }(\%)^{\star}\end{array}$ & $\begin{array}{l}\text { Número de } \\
\text { óbitos de causas } \\
\text { mal definidas } \\
\text { reclassificados } \\
\text { no relacionamento } \\
\text { com SIH (N) }\end{array}$ & $\begin{array}{l}\text { Mortalidade } \\
\text { proporcional nos } \\
\text { óbitos de causas } \\
\text { mal definidas } \\
\text { reclassificados no } \\
\text { relacionamento, } \\
\text { incluindo mal } \\
\text { definidas (\%) }\end{array}$ & $\begin{array}{l}\text { Mortalidade } \\
\text { proporcional após } \\
\text { reclassificação dos } \\
\text { óbitos de causas } \\
\text { mal definidas, } \\
\text { excluindo os } \\
\text { resíduos } \\
\star \star, \star \star \star ~(\%) ~\end{array}$ \\
\hline Aparelho circulatório & 34.246 & 29,7 & 33,4 & 772 & 36,2 & 33,4 \\
\hline Causas externas & 15.822 & 13,7 & 15,4 & 116 & 5,4 & 15,2 \\
\hline Neoplasias & 14.293 & 12,4 & 13,9 & 245 & 11,5 & 13,9 \\
\hline Aparelho respiratório & 12.694 & 11,0 & 12,4 & 287 & 13,5 & 12,4 \\
\hline Mal definidas & 12.633 & 11,0 & & 77 & 3,5 & \\
\hline Perinatais & 3.397 & 2,9 & 3,3 & 6 & 0,3 & 3,2 \\
\hline $\begin{array}{l}\text { Endócrinas, nutricionais } \\
\text { e metabolólicas }\end{array}$ & 6.511 & 5,6 & 6,3 & 222 & 10,4 & 6,4 \\
\hline Infecciosas e parasitárias & 5.720 & 5,0 & 5,6 & 149 & 7,0 & 5,6 \\
\hline Aparelho digestivo & 4.552 & 3,9 & 4,4 & 135 & 6,3 & 4,5 \\
\hline Geniturinárias & 1.856 & 1,6 & 1,8 & 38 & 1,8 & 1,8 \\
\hline Neurológicas & 1.116 & 1,0 & 1,1 & 23 & 1,1 & 1,1 \\
\hline Malformações & 850 & 0,7 & 0,8 & 11 & 0,5 & 0,8 \\
\hline Hematológicas, imunitárias & 539 & 0,5 & 0,5 & 13 & 0,6 & 0,5 \\
\hline Transtornos mentais & 392 & 0,3 & 0,4 & 8 & 0,4 & 0,4 \\
\hline Pele e tecido subcutâneo & 251 & 0,2 & 0,2 & 7 & 0,3 & 0,2 \\
\hline Reumatológicas & 227 & 0,2 & 0,2 & 10 & 0,5 & 0,2 \\
\hline Parto, gravidez e puerpério & 202 & 0,2 & 0,2 & 14 & 0,7 & 0,2 \\
\hline Otorrinolaringológicas & 10 & 0,0 & 0,0 & 0 & 0,0 & 0,1 \\
\hline Oftalmológicas & 1 & 0,0 & 0,0 & 0 & 0,0 & 0,0 \\
\hline Total & 115.312 & 100,0 & 100,0 & 2.133 & 100,0 & 100,0 \\
\hline
\end{tabular}

* Total de óbitos: 115.312-12.633;

** Total de óbitos: $(115.312-12.633)+(2133-77)$

*** Resíduos: 77 mal definidos.

Porém, se pudéssemos inferir a reclassificação feita na fração daqueles que conseguimos relacionar com as AIH do SIH-SUS para o conjunto de todos os óbitos de causa mal definida, seríamos capazes de observar alterações relevantes nas taxas de mortalidade específicas, por idade, para alguns capítulos da CID-10, especialmente os capítulos de doenças crônicas. Como exemplo, nas "Doenças do Aparelho Circulatório", a taxa de mortalidade na faixa de quarenta anos ou mais, sem incluir os óbitos de causa mal definida reclassificados, seria elevada de 76,9 por $10 \mathrm{mil}$, para 87,5 por $10 \mathrm{mil}$, ao incluir estes óbitos. Para esse cálculo, foi necessário obter o tamanho da população específica, isto é, de quarenta anos ou mais, no Estado do Rio de Janeiro, em 1998. Portanto, o grande número de casos de óbitos de causa mal definida pode distorcer as séries temporais das taxas de mortalidade específicas para determinados grupos de causas.

Em geral, estudos sobre mortalidade proporcional descartam os óbitos de causa mal definida 2. Embora a falta de uma causa básica do óbito tenha sido o critério de inclusão do caso no presente estudo, esta não foi a única variável com informação escassa ou incompleta. Pôde-se observar que as variáveis estudadas apresentaram graus variados de completude. Repare-se que apenas $58,5 \%$ das DO tinham registro de escolaridade, e até mesmo a informação sobre quem identificou o óbito teve uma completude de apenas de 79,6\%.

Os casos de óbito sem causa básica definida apresentaram características que os distinguiam daqueles com causas definidas. Predominaram os adultos em idade produtiva, com baixa escolaridade, cor da pele não branca, vi- 
vendo na área metropolitana, cujo óbito ocorreu sem assistência médica ou que foram atendidos com maior frequência em algumas unidades de saúde do sistema público, aonde chegaram, por vezes, já cadáveres.

Se os percentuais de óbitos de causa mal definida e óbitos de causa definida ocorridos em hospitais não divergem muito, o percentual de coincidência dos óbitos de causa mal definida com internação que gerou AIH foi notável e significativamente menor do que aqueles dos óbitos de causa definida. Nos óbitos de causa mal definida, esta coincidência ocorreu em apenas cerca de um quarto dos relacionamentos, enquanto que nos óbitos de causa definida este índice foi de quase dois terços. Portanto, é provável que grande parte dos óbitos de causa mal definida hospitalizados tenham ocorrido em emergências, sem gerar AIH.

Reis 3, no período de 1980 a 1995, analisou a distribuição e as características dos óbitos de causa mal definida na Região Metropolitana do Rio de Janeiro. Entre os nossos achados e os de Reis 3 , as diferenças são irrelevantes quanto à distribuição da freqüência da assistência médica, local de ocorrência do óbito, faixa etária e gênero dos óbitos de causa mal definida.

Em estudo desenvolvido na Cidade do Rio de Janeiro, em 2001, com base nas causas dos óbitos de causa mal definidas, estimadas a partir de dados de prontuários hospitalares, visitas domiciliares, registros do Instituto Médico Legal e boletins de ocorrência da polícia, verificou-se que, dos 1.531 casos investigados, foi possível identificar a causa do óbito em $49,3 \%$, dentre os quais $33,1 \%$ se deveram a morte violenta 16. A comparação desses resultados com os do presente estudo é prejudicada pelo uso de bases de dados e métodos de pareamento diferentes, além das diferenças de população, faixa etária, região e época. Na Cidade do Rio de Janeiro, as autoras encontraram uma freqüência mais elevada de mortes violentas nos óbitos de causa mal definida, enquanto em nosso estudo isso ocorreu com doenças crônicas.

Santos \& Noronha 17 calcularam as taxas de mortalidade em conglomerados de bairros do Município do Rio de Janeiro, agrupados por fatores sócio-econômicos (renda familiar e condições de saneamento), conseguindo identificar relações entre padrões de mortalidade e nível sócio-econômico. O estudo mostrou que, nos bairros de população mais abastada, a taxa de mortalidade por "Doença do Aparelho Circulatório" era mais baixa que a taxa média do município, ao passo que, nos bairros mais pobres, a taxa de óbitos de causa mal definida era mais alta. Houve concordância com os resulta- dos encontrados no nosso estudo, pois, entre os óbitos de causa mal definida, o grau de instrução tendeu a ser mais baixo.

No Brasil, em 1995, entre os 95.446 óbitos sem causa definida, $65 \%$ ocorreram sem assistência médica 18. Essa assistência refere-se ao momento no qual ocorre o óbito, não significando necessariamente falta de assistência no período que o antecede. No Rio de Janeiro, em 1998 , este percentual foi de $54,8 \%$. Mesmo que essa informação não reflita a real cobertura da assistência médica, ainda assim não foi suficiente para esclarecer as causas das mortes.

Chama a atenção o fato de que, em cinco regiões de saúde, o percentual de relacionamento dos óbitos de causa mal definida com as SIH-SUS foi superior a $25 \%$. O percentual médio desses relacionamentos foi baixo no Estado como um todo, em virtude do baixo percentual na região metropolitana, onde também o percentual de relacionamento dos óbitos de causa definida foi o menor. O problema é agravado ainda mais pelo fato de que quase a metade dos óbitos de causa mal definida ocorreu em apenas dez unidades de saúde, todas localizadas na região metropolitana. Portanto, na região onde ocorreram quase quatro em cada cinco óbitos, a metropolitana, as possibilidades de relacionamento de informações de DO e SIH-SUS são modestas, independentemente do registro da causa, o que pode estar relacionado com o perfil das causas e com a estrutura de atenção à demanda da população.

Comparando-se o cálculo da mortalidade proporcional que exclui os óbitos mal definidos com o que inclui os óbitos reclassificados neste estudo, constatamos variações modestas. Esta correção não modificou a ordem de importância dos capítulos. A metodologia empregada para a reclassificação dos casos de óbitos de causa mal definida não evitou que 77 dos óbitos relacionados continuassem sem causa definida provável.

Comparando-se a mortalidade proporcional por capítulo da CID-10 nos 2.133 casos de óbitos de causa mal definida reclassificados com aquela em que se descartaram os óbitos de causa mal definida, percebemos que a mortalidade proporcional do capítulo das "Doenças do Aparelho Circulatório” é maior nos óbitos de causa mal definida reclassificados.

Oliveira et al. ${ }^{4}$, estudando a evolução da mortalidade por doenças do aparelho circulatório, entre 1980 e 2000, observaram declínio mais acentuado das taxas de mortalidade por doenças isquêmicas do coração no Estado do Rio de Janeiro, coincidente com o aumento das taxas por óbitos de causa mal definida. 
Em princípio, não esperaríamos reclassificar os óbitos de causa mal definida como causa externa, uma vez que os de causas externas têm indicação de exame médico-legal, resultando em definição da causa do óbito. Ainda assim, reclassificamos $5,4 \%$ dos óbitos de causa mal definida no capítulo das causas externas.

A grande dificuldade deste trabalho consistiu no fato de não existir identificador único que permita relacionar óbitos da DO com internações hospitalares, registradas nas SIH-SUS.

Chama-se atenção para o fato de que o SIHSUS não é suficiente para a investigação das causas de todos os óbitos de causa mal definida, uma vez que boa parte deles ocorrem em hospitais não vinculados ao SUS. Essa limitação é intransponível, uma vez que o SIH-SUS é a única grande base de dados hospitalares existente.

É importante destacar que o preenchimento adequado da DO não pode ser substituído por qualquer processo de relacionamento probabilístico entre SIH-SUS e DO. Os dois documentos, DO e SIH-SUS, têm finalidades e princípios diferentes, o que fica evidente nos baixos índices de concordância entre as causas básicas de óbito na DO e o diagnóstico principal no SIH-SUS. Se fossemos comparar o diagnóstico principal no SIH-SUS com a causa básica de óbito na DO com detalhamento de quatro dígitos, as diferenças poderiam ser ainda mais notáveis.
Os resultados aqui encontrados referem-se ao Estado do Rio de Janeiro, no ano de 1998, e não podem ser generalizados para outros locais e períodos. Podemos concluir que a proporção de óbitos por causa mal definida está relacionada com desigualdades no acesso e na atenção médica prestada, uma vez que é maior em mulheres, em indivíduos de cor da pele não branca de baixa escolaridade, atendidos em grandes unidades públicas da periferia da região metropolitana.

A qualidade das informações em saúde deve ser melhorada para que a utilização destas possa subsidiar a implementação de ações voltadas para o aperfeiçoamento da atenção à saúde. Essa melhora pode ser obtida estimulandose as escolas médicas a incluir no currículo disciplinas sobre sistemas de informação em saúde e concentrando esforços na monitorização da qualidade da informação nas unidades de saúde com maior volume de problemas na identificação das causas de óbito.

É necessário, também, estimular a disseminação de serviços de verificação de óbitos com respaldo legal, para permitir a realização de autópsia sempre que a causa de óbito for indeterminada. Além disso, é preciso revisar normas e condutas no preenchimento do atestado de óbito para estimular os médicos a procurarem mais informações sobre os processos que levam ao óbito.

\section{Resumo}

Este artigo descreve características sócio-demográficas e clínicas dos óbitos classificados como de causa mal definida, ocorridos no Estado do Rio de Janeiro, Brasil, em 1998, e faz a reclassificação dos grupos de causas prováveis desses óbitos, com base nas informações contidas nas Declarações de Óbito do Sistema de Informações de Mortalidade e do Sistema de Informações Hospitalares do Sistema Único de Saúde (SIHSUS) de 1997 e 1998. Para isso, foi necessário relacionar uma amostra aleatória de óbitos de causas definidas com o SIH-SUS. Foi utilizada técnica de relacionamento probabilístico de registros. Comparando com os óbitos de causa definida, os óbitos de causa mal definida ocorreram mais em indivíduos de cor da pele não branca e com menor escolaridade; foram mais frequentes na região metropolitana e os indivíduos internaram menos no SUS e morreram mais em domicílios, e sem assistência médica. Foi possível reclassificar cerca de $20 \%$ dos óbitos de causa mal definida. A reclassificação apresentou pequeno impacto na mortalidade proporcional, por causa do tamanho deste grupo (10\% dos óbitos). No entanto, se os resultados pudessem ser aplicados ao total de óbitos de causa mal definida, poderiam ocorrer alterações de maior impacto na mortalidade proporcional.

Sistemas de Informação; Registros de Mortalidade; Atestados de Óbito 


\section{Colaboradores}

Os autores participaram ativamente de todas as fases do estudo. C. L. S. Teixeira participou de todas as etapas: revisão da literatura, desenho do estudo, processamento dos dados, análises estatísticas, interpretação e discussão dos resultados e redação final do artigo. K. V. Bloch e C. H. Klein contribuíram na revisão da literatura, planejamento do desenho do estudo, análises estatísticas, interpretação e discussão dos resultados e redação final do artigo. C. M. Coeli colaborou no processo de relacionamento dos bancos, com a interpretação e discussão dos resultados, além da redação final do artigo.

\section{Referências}

1. Vasconcelos AMN. Estatísticas de mortalidade por causas: uma avaliação da qualidade da informação. In: Anais do X Encontro de Estudos Populacionais. v. 1. Rio de Janeiro: Associação Brasileira de Estudos Populacionais; 1996. p. 151-66.

2. Laurenti R. Mortalidade Brasil - 2000. In: Departamento de Informação e Informática do SUS, Ministério da Saúde. Sistema de Informação Sobre Mortalidade (SIM) 1996 a 2000 [CD-ROM]. Brasília: Departamento de Informação e Informática do SUS, Ministério da Saúde; 2000.

3. Reis ACGV. Mortalidade por causas mal definidas na Região Metropolitana do Rio de Janeiro, de 1980 a 1995 [Dissertação de Mestrado]. Rio de Janeiro: Escola Nacional de Saúde Pública, Fundação Oswaldo Cruz; 1998.

4. Oliveira GMM, Klein CH, Silva NAS. Mortalidade por doenças cardiovasculares em três estados do Brasil de 1980 a 2002. Rev Panam Salud Pública 2006; 19:85-93.

5. Secretaria Estadual de Saúde do Rio de Janeiro. Portaria n. 550. Diário Oficial do Estado do Rio de Janeiro 1990; 26 jan.

6. Teixeira CLS. Relacionamento e reclassificação dos óbitos de causa mal definida com as autorizações de internação hospitalar no Sistema Único de Saúde, no Rio de Janeiro, em 1998 [Dissertação de Mestrado]. Rio de Janeiro: Núcleo de Estudos de Saúde Coletiva, Universidade Federal do Rio de Janeiro; 2004.

7. Teixeira CLS, Bloch KV, Klein CH, Coeli CM. Método de relacionamento de banco de dados do Sistema de Informações sobre Mortalidade (SIM) e das autorizações de internação hospitalar (BDAIH) no Sistema Único de Saúde (SUS), na investigação de óbitos de causa mal definida no Estado do Rio de Janeiro, 1998. Epidemiol Serv Saúde 2006; 15:47-57.

8. Coeli CM. Vigilância do diabetes mellitus em uma população idosa: aplicação da metodologia de captura-recaptura [Tese de Doutorado]. Rio de Janeiro: Instituto de Medicina Social, Universidade do Estado do Rio de Janeiro; 1998.
9. Mathias TAF, Soboll MLMS. Confiabilidade de diagnósticos nos formulários de autorização de internação hospitalar. Rev Saúde Pública 1998; 32: 526-32.

10. Melo ECP. Infarto agudo do miocárdio no Município do Rio de Janeiro: qualidade dos dados, sobrevida e distribuição espacial [Tese de Doutorado]. Rio de Janeiro: Escola Nacional de Saúde Pública, Fundação Oswaldo Cruz; 2004.

11. Soares EP. Associação entre peso ao nascer e mortalidade infantil no Município de Campos de Goytacazes [Dissertação de Mestrado]. Rio de Janeiro: Núcleo de Estudos de Saúde Coletiva, Universidade Federal do Rio de Janeiro; 2003.

12. Machado CJ. A literature review of record linkage procedures focusing on infant health outcomes. Cad Saúde Pública 2004; 20:362-71.

13. Organização Mundial da Saúde. Classificação estatística de doenças e problemas relacionados à saúde - 10a revisão. São Paulo: Edusp; 1996.

14. Sistema de Vigilância Sanitária, Ministério da Saúde. Sistema de Informações sobre Mortalidade SIM. http://www.datasus.gov.br (acessado em 02/ Abr/2004).

15. Fleiss JL. Statistical methods for rates and proportions. New York: Witez; 1981.

16. Caridade MC, Campos TC, Serafim FC, Bellizzi ALM, Simplício AMB, Theme-Filha MM, et al. Causas mal definidas de morte: recuperação de informações na Cidade do Rio de Janeiro. Revista Saúde em Foco 2001; 22:63-73.

17. Santos SM, Noronha CP. Padrões espaciais de mortalidade e diferenciais sócio-econômicos na cidade do Rio de Janeiro. Cad Saúde Pública 2000; 17:1099-110.

18. Vermelho L, Costa AJL, Kale PL. Indicadores de saúde. In: Medronho RA, Carvalho DM, Luiz RR, Werneck GL, organizadores. Epidemiologia. São Paulo: Editora Atheneu; 2002. p. 33-55.

Recebido em 17/Fev/2005

Versão final reapresentada em 24/Out/2005

Aprovado em 19/Dez/2005 